\title{
Emergent Tricuspid Valve Replacement for Tricuspid Valve Endocarditis in a Chronic Hemodialysis Patient in Suriname
}

\author{
Rosita Bihariesing $h^{1 *}$, Pieter Voigt ${ }^{2}$, Juliett Garcia ${ }^{2}$ and Soeradj Harkisoen ${ }^{3}$ \\ ${ }^{1}$ Department of Anaesthesiology, Academic Hospital Paramaribo, Republic of Suriname \\ ${ }^{2}$ Department of Cardiology and Cardiothoracic Surgery, Academic Hospital Paramaribo, Republic of Suriname \\ ${ }^{3}$ Department of Internal Medicine and Infectious Diseases, Academic Hospital Paramaribo, Republic of Suriname
}

\begin{abstract}
Tricuspid valve endocarditis caused by Acinetobacter baumannii is associated with a high mortality rate because of the bacterium's resistance to almost all available antibiotics. Low and middle income countries (LMICS) face the challenge of successfully treating infective endocarditis because of the delayed diagnosis and having little access to expensive drug regimens. Although several international guidelines indicate that large vegetations on cardiac valve protheses require immediate surgery for valve replacement, this intervention remains a challenge in LMICs with limited resources. In our case, a hemodialysis patient developed tricuspid valve endocarditis due to $A$. Baumannii which was diagnosed at a late stage when surgery was necessary. Despite a few major complications, the patient survived. At the 2-year follow-up, she is still doing well.
\end{abstract}

Keywords

Endocarditis, Acinetobacter baumannii, Tricuspid valve

\section{Introduction}

Infective endocarditis (IE) in patients with end-stage renal disease continues to increase worldwide, with high morbidity and mortality up to $30-63 \%$ during the first year after diagnosis $[1,2]$. Tricuspid valve (TV) IE accounted for $5 \%-15 \%$ of all IE cases [2]. Acinetobacter baumannii, a rare cause of IE in native and prosthetic heart valves [3], is characterized by an acute onset and aggressive course. Mortality tends to be higher in patients with native- than prosthetic-valve endocarditis because of the low index of suspicion, leading to delayed treatment [3]. We report a hemodialysis patient with TVIE due to $A$. baumannii who presented at a late stage and required emergency valve replacement. This case is important because treating IE is challenging in low and middle income countries (LMICs), with limited healthcare facilities [4].

\section{Case Presentation}

A 26-year-old woman on dialysis had several episodes of dialysis-catheter infections with an Acinetobacter baumannii bacteremia, susceptible to amoxicillin/clavulanic acid. She underwent repeated dialysis-catheter replacements and antibiotic treatments. Due to the recurrent nature of the infections, meropenem instead of amoxicillin/clavulanic acid was administered in the most recent prior admission to cover possible multi-resistant microorganisms. She was now admitted with fever, chills, dyspnea, cough, generalized muscle pains, and anasarca. Laboratory test results (Table 1 ) revealed a leukocy- tosis, anemia, and elevated serum C-reactive protein. Serum electrolytes and liver tests were unremarkable. Chest radiography showed enhanced bilateral vascular distribution, cardiomegaly, and a consolidation in the right lung with pleural effusion. Blood cultures were positive for $A$. baumannii (with the same susceptibility as previously), and meropenem was started for aforementioned reasons. Transthoracic echocardiography showed a large $(10.6 \times 36.5 \mathrm{~mm})$ vegetation on the tricuspid valve (TV) (Figure 1), severe TV regurgitation (Figure 2 ), and right ventricular failure. Because of the right heart failure and large vegetation, patient was accepted for surgery.

Infected tissue was resected and the TV was replaced with a $27 \mathrm{~mm}$ mechanical prosthesis. Transesophageal echocardiography assessed a good tricuspid valve prosthesis function, moderate function of the right ventricle, and good function of the left ventricle. The patient came off bypass with high-dose

*Corresponding author: Rosita Bihariesingh, Department of Anaesthesiology, Academic Hospital Paramaribo, 1-3 Flustraat, Paramaribo, Suriname, Tel: $+44-2222$ (ext 601)

Accepted: December 12, 2020

Published online: December 14, 2020

Citation: Bihariesingh R, Voigt P, Garcia J, et al. (2020) Emergent Tricuspid Valve Replacement for Tricuspid Valve Endocarditis in a Chronic Hemodialysis Patient in Suriname. J Cardiothorac Surg Ther 4(1):65-67 
Citation: Bihariesingh R, Voigt P, Garcia J, et al. (2020) Emergent Tricuspid Valve Replacement for Tricuspid Valve Endocarditis in a Chronic Hemodialysis Patient in Suriname. J Cardiothorac Surg Ther 4(1):65-67

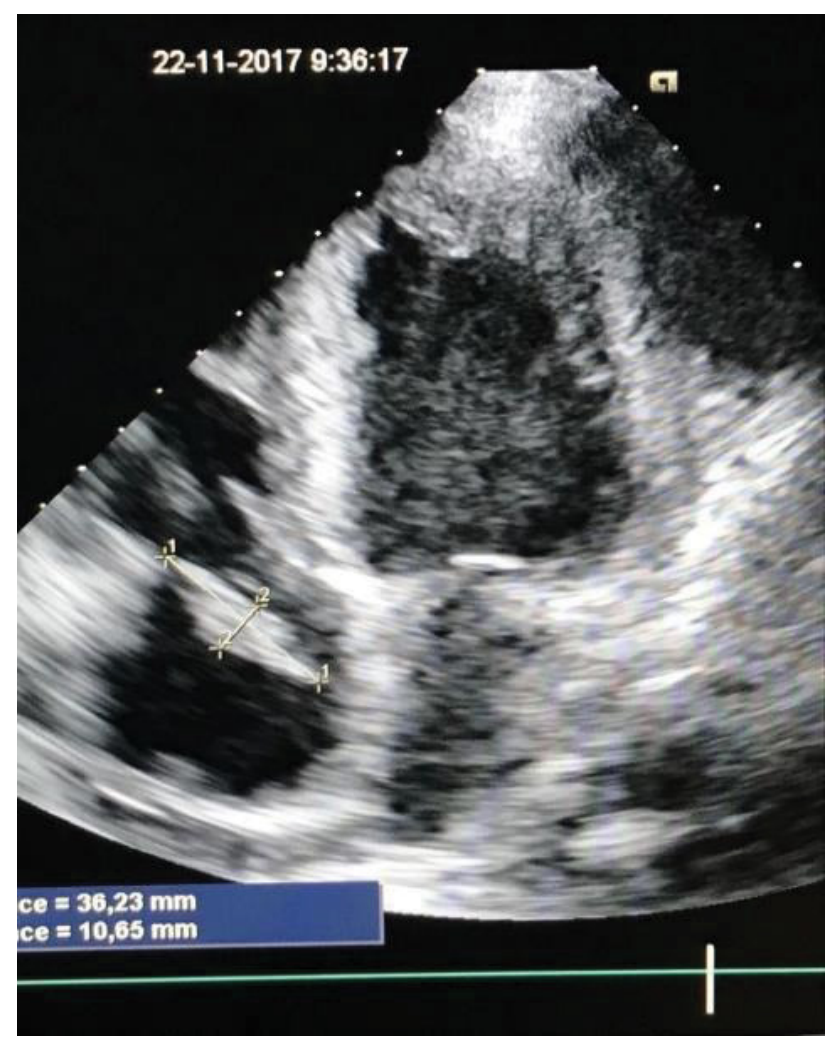

Figure 1: Echocardiography of the patient, there is a large vegetation on the tricuspid valve.

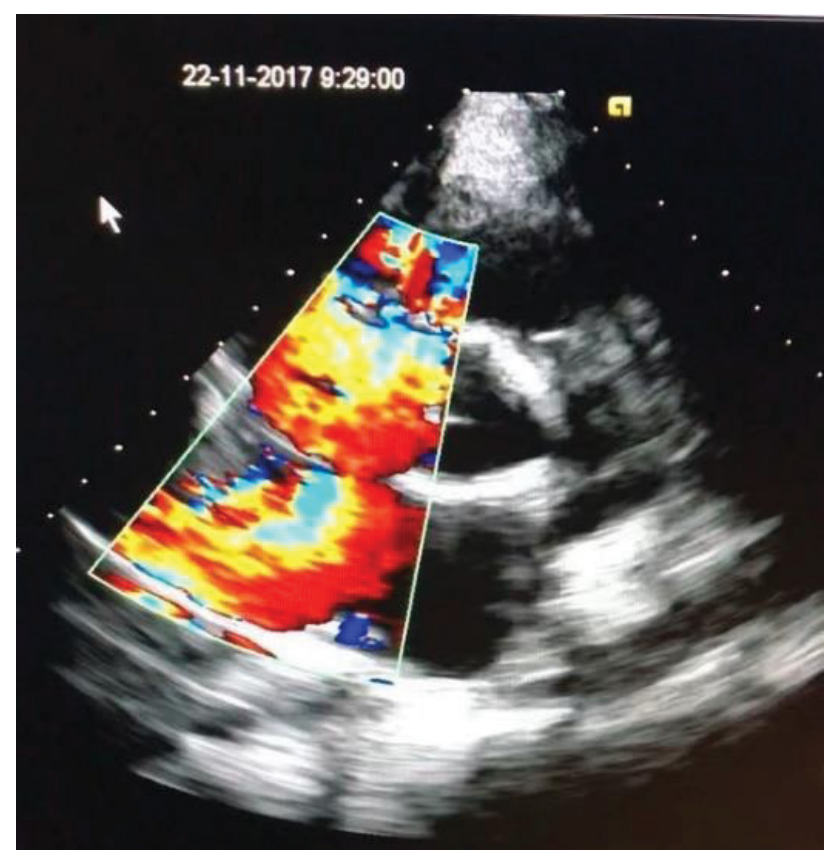

Figure 2: Severe tricuspid valve regurgitation is apparent.

inotropic support. On postoperative day (POD) 3, hemodialysis was restarted, without complications. The postoperative course was complicated by cardiac arrest and two episodes of cardiac tamponade and bleeding, each time requiring re-exploration. Cultures of the removed TV and vegetations were positive for $A$. baumannii, with the same antibiotic resistance
Table 1: Laboratory findings during the patient's hospitalization for tricuspid valve infective endocarditis.

\begin{tabular}{|l|l|l|l|}
\hline Laboratory test & Day 0 & Day 14 & Day 45 \\
\hline $\mathrm{Hb}(\mathrm{mmol} / \mathrm{L})$ & 4.5 & 4.3 & 5.2 \\
\hline Leukocytes $\left(\times 10^{9} / \mathrm{L}\right)$ & 31.8 & 40.5 & 8 \\
\hline BUN $(\mathrm{mmol} / \mathrm{L})$ & 10.7 & 10.7 & 3.3 \\
\hline Creatinine $(\mathrm{mmol} / \mathrm{L})$ & 345 & 384 & 291 \\
\hline CRP $(\mathrm{mg} / \mathrm{dL})$ & 22.7 & 17.9 & 4.7 \\
\hline
\end{tabular}

BUN: Blood urea nitrogen, CRP: C-reactive protein, $\mathrm{Hb}$ : Hemoglobin

patterns as in previous blood cultures. Meropenem was continued for 6 weeks. Additionally, she developed a ventilator-associated pneumonia with $A$. baumannii, which was resistant to every intravenous antibiotic available in our country. Nebulization with colistin was started, with good results. The patient was extubated on POD 17. On POD 22, a bilateral pneumothorax was treated with chest drains. On POD 32, she transferred to the cardiac ward for further recovery. On POD 45 she was discharged home in good clinical condition with normalized infection parameters (Table 1). Currently, nearing 2-years and 8 months follow-up, she is in good clinical condition, undergoing dialysis using a Cimino shunt.

\section{Discussion}

Infective endocarditis remains a disease with high morbidity and mortality rates, even in high-income countries. Treatment of IE in MIC's has several additional challenges, related to the limited access to health care, lack of availability of medical equipment and medications, high prevalence of multi-resistant pathogens, and economic inequalities [5]. Endocarditis patients in MIC's often present late after initiation of symptoms [3]. Consequently, patients have frequently already developed complications (e.g. heart failure, valve abscess, septic emboli) resulting in a worsened prognosis [3]. Our patient presented with RV failure and a large vegetation on her tricuspid valve, surgery was indicated instead of antibiotic treatment alone. Acetinobacter baumanii is an opportunistic pathogen, known for its high rate of antibiotic resistance and high prevalence in nosocomial infections. In IE, A. baumannii has a high mortality rate up to $56 \%$ [3]. Its antibiotic resistance, combined with the little access to expensive new generation antibiotics, provides an extra challenge in treating infections caused by $A$. baumanii in MIC's. Furthermore, missed drug doses or unnecessary regimen changes due to antibiotic 'stock-outs' may also occur. In conclusion, this report shows that surgery for complicated TVIE with a multi-resistant pathogen is possible in MIC's, despite several challenges that need to be overcome. Patients in MIC's presenting with complex IE therefore should not be declined the option of surgery.

\section{Conflicts of Interest}

None declared.

\section{Funding}

None. 


\section{Authors Contribution}

$\mathrm{RB}, \mathrm{PV}, \mathrm{JG}$, and SH performed the clinical work and contributed to writing the manuscript. All authors have read and approved the final manuscript.

\section{Consent}

Written informed consent was obtained from the patient for publication of this case report and accompanying images. A copy of the written consent is available.

\section{References}

1. Baroudi S, Qazi RA, Lentine L, et al. (2008) Infective endocarditis in haemodialysis patients: 16 -year experience at one institution. NDT Plus 1: 253-256.

2. Kamalakannan D, Pai RM, Johnson LB, et al. (2007) Epidemiology and clinical outcomes of infective endocarditis in hemodialysis patients. Ann Thorac Surg 83: 2081-2086.

3. Chen Q, Cao H, Lu H, et al. (2015) Bioprosthetic tricuspid valve endocarditis caused by Acinetobacter baumannii complex, a case report and brief review of the literature. J Cardiothoracic Surg 10: 149.

4. Njuguna B, Gardner A, Karwa R, et al. (2017) Infective endocarditis in low and middle-income countries. Cardiol Clin 35: 153-163.

5. Choudhary SK, Talwar S, Airan B (2016) Choice of prosthetic heart valve in a developing country. Heart Asia 8: 65-72. 